\title{
A search for new variable stars in NGC 6231^
}

\author{
T. Arentoft ${ }^{1, \star \star}$, C. Sterken ${ }^{1, \star \star \star}$, M. R. Knudsen ${ }^{1}$, L. M. Freyhammer ${ }^{1,2}$, H. W. Duerbeck ${ }^{1}$, \\ E. Pompei ${ }^{3}$, C. E. Delahodde ${ }^{3}$, and J. W. Clasen ${ }^{4}$ \\ 1 University of Brussels (VUB), Pleinlaan 2, 1050 Brussels, Belgium \\ 2 Royal Observatory of Belgium, Ringlaan 3, 1180 Brussels, Belgium \\ 3 European Southern Observatory, Alonso de Cordova 3107, Santiago, Chile \\ 4 Nordic Optical Telescope, Apartado 474, 38700 Santa Cruz de La Palma, Canary Islands, Spain
}

Received 27 September 2001 / Accepted 15 October 2001

\begin{abstract}
NGC6231 is a well-studied young open cluster hosting several variable stars. In the field six $\beta$ Cephei stars, several eclipsing binaries and one $\delta$ Scuti star are known (as well as a foreground $\delta$ Scuti star). In an ongoing effort to map and study the variability in NGC 6231, we present new results based on CCD time-series data optimised for the bright $\beta$ Cephei stars as well as data optimised for much fainter stars. We detect 17 new variable stars in the cluster, including three $\delta$ Scuti stars, three $\gamma$ Doradus candidates, three Slowly Pulsating B star candidates and one, or possibly two, new $\beta$ Cephei stars.
\end{abstract}

Key words. stars: variables: general - individual: NGC 6231 - techniques: photometric - methods: data analysis

\section{Introduction}

The present paper is part of an extensive effort to study short-period variable stars in the young open cluster NGC 6231. This includes searching for new and confirming known variable stars and monitoring the long-term stability of the variations - i.e. investigate amplitude and frequency variations in a rich set of variable stars of different types. The synopsis of this project is given by Sterken et al. (2002).

NGC 6231 has been intensively studied in the past decades, as recently reviewed by Sung et al. (1998, hereinafter SBL). They carried out $U B V R I$ and $\mathrm{H} \alpha$ photometry for a number of stars in the cluster in order to determine cluster parameters and search for pre-main sequence (PMS) stars. Baume et al. (1999, hereinafter BVF) carried out a similar photometric $U B V I$ study and the results were found to be in excellent agreement with SBL. BVF found an age of the cluster of 3-5 Myr.

Six $\beta$ Cephei stars are known in the cluster (Balona \& Shobbrook 1983, BS83; Balona \& Engelbrecht 1985, BE85). Balona \& Laney (1995, BL95) searched for

Send offprint requests to: T. Arentoft,

e-mail: tarentof@vub.ac.be

* Based on observations obtained at the Danish 1.54-m and Dutch 0.9-m telescopes at ESO (ESO applications: 60D-0148, $61 \mathrm{D}-0128$ and $62 \mathrm{H}-0110)$.

$\star \star$ Now at the Royal Observatory of Belgium.

$\star \star \star$ Research Director, Belgian Fund for Scientific Research (FWO). additional short-period variables and found one foreground and one possible cluster member $\delta$ Scuti star and 3 eclipsing variables. No additional pulsating B-stars were found ( $\beta$ Cephei or Slowly Pulsating B (SPB) stars). Furthermore, a number of spectroscopic-binary stars have been found in the cluster (García \& Mermilliod 2001; Raboud 1996; Levato \& Morrell 1983).

Finding new pulsating stars of different types in NGC 6231 (or any open cluster) is important, as it allows for comparative studies of different classes of pulsating stars originating from common initial conditions.

In this paper we present the results of a search for new variable stars in NGC 6231 using CCD time-series data. A part of the observations was optimised for the comparatively bright $\left(\sim 9^{\mathrm{m}}\right) \beta$ Cephei stars and another part for stars of $13^{\mathrm{m}}-14^{\mathrm{m}}$. The latter part allows us to search for possible cluster $\delta$ Scuti and $\gamma$ Doradus $^{1}$ stars, while in those "deep" data, the $\beta$ Cephei stars are, naturally, overexposed. We discuss the newly detected variable stars individually and give tentative frequency solutions as indicators of the time scales and amplitudes of the variations.

\section{The data and data reduction}

Data were obtained at ESO during 6 individual observing runs in 1998-2000, as outlined in Table 1 . The observations in 1998-1999 were optimised for the fainter stars

\footnotetext{
1 A new class of pulsating variables, known since Cousins \& Warren's (1963) suggestion of having found photometric variability in $\gamma$ Doradus, the prototype of the class.
} 
Table 1. Data sets discussed in this paper. D90 is the Dutch 0.90-m telescope, D154 the Danish 1.54-m telescope, both at ESO, La Silla, Chile. Comment deep indicates that the observations were optimised for the fainter stars of the cluster, as discussed in the text.

\begin{tabular}{llllrrrl}
\hline Run & HJD & Observer & Telescope & \#nights & \#useful images & filters & comment \\
\hline 1998 March & $2450887-891$ & CS & D90 & 4 & 62 & $V$ & deep \\
1998 April & $2450918-922$ & TA & D90 & 5 & 277 & $V$ & deep \\
1998 June & $2450970-985$ & LF, JC & D154 & 10 & 921 & $V$ & deep \\
1999 March & $2451263-269$ & CS & D90 & 4 & 150 & $V$ & deep \\
2000 June & $2551721-722$ & HD & D154 & 2 & 240 & $u v b y$ & \\
2000 July & $2451737-744$ & EP, CD & D154 & 7 & 1050 & uvby & \\
\hline \hline
\end{tabular}

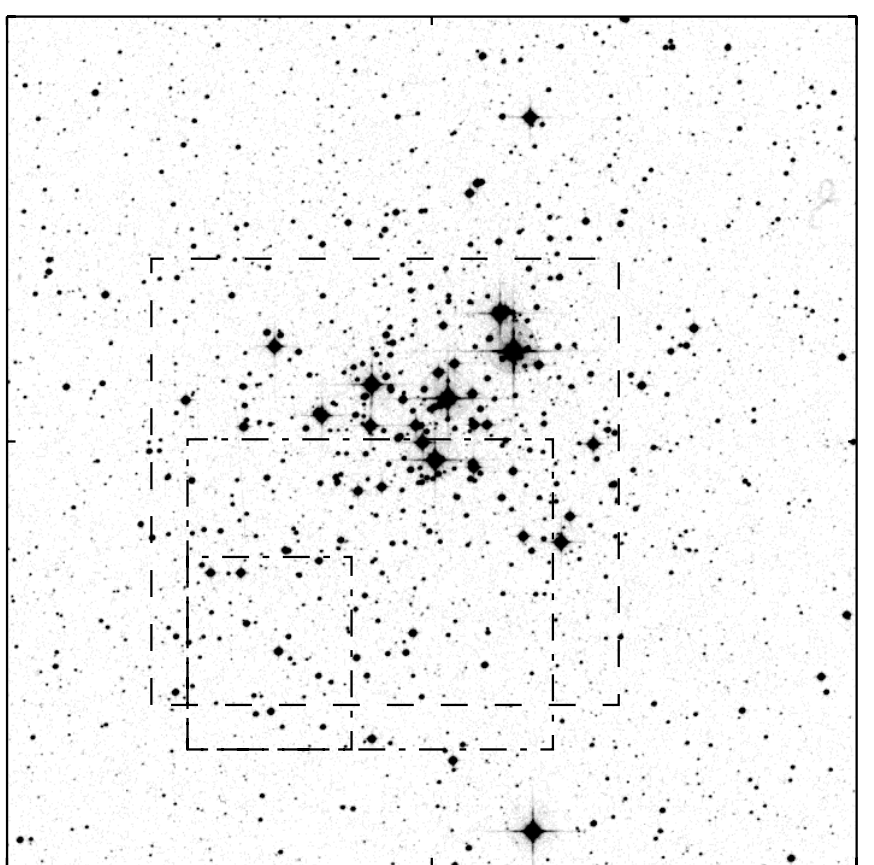

Fig. 1. Digitized sky survey image of NGC $6231\left(20^{\prime} \times 20^{\prime}, \mathrm{N}\right.$ is up, $\mathrm{E}$ is to the left). The three superimposed boxes show the fields covered by our observations. The smallest field was observed with the Dutch 0.9-m telescope (ESO), the mediumsize field was observed with the Danish 1.54-m telescope (ESO) in 1998 June and the large field was observed in 2000 June and July, also with the Danish 1.54-m. See Table 1 for further details.

$\left(13^{\mathrm{m}}-14^{\mathrm{m}}\right)$ while the observations in 2000 were optimised for the several magnitudes brighter $\beta$ Cephei stars. Two different ESO telescopes were used, the Dutch 0.9-m (D90) and the Danish 1.54-m (D154). Three overlapping fields of different sizes were covered by the time-series observations, as shown in Fig. 1.

The data obtained at the D90 were bias-corrected and flat fielded using (standard) techniques described in e.g. Arentoft \& Sterken (2000). Observations were made in the $V$ passband and covered about 2 hours of time series during 13 individual nights.

Two output amplifiers were used for the CCD readout at the D154 in 1998 June and also in this case only the $V$-filter was used. The time was shared with another programme and only during one night was a time string longer than 6 hours collected. Significant pointing offsets from night to night imply that some stars are only present on some of the frames. As the severe overexposure of the bright stars caused charge bleeding on the CCD, a correction for cross-talk between the two amplifiers had to be applied. Because the number of useful $V$ sky-flats from the individual nights was limited, we used one combined (median) master flat for the whole run. Science and flatfield images were corrected for the amplifier cross-talk following Freyhammer et al. (2002). Then bias subtraction was made by subtracting constants measured in the overscan regions and finally the science images were corrected for gain variations using the combined flatfield.

The observations in 2000 June and July with the D154 were done in uvby. Also in this case, significant pointing offsets occurred from night to night. Owing to bad weather during parts of the two runs, relatively few flatfields in each filter were collected. We therefore combined the $y$ flatfields from one good night of both runs and created master flats for June and July, respectively. For $u v$ and $b$, we created one master flat in each filter and applied it to both the June and July data. The images did not contain an overscan strip so the zero-point levels were estimated from evening, night and morning BIAS frames. A linear change in the zero-point level during the night was found and corrected for.

The photometric reductions were carried out in all cases using MOMF (Kjeldsen \& Frandsen 1992). This very robust, semi-automatic program combines PSF and aperture photometry, uses a local sky-background determination and calculates differential magnitudes relative to a weighted mean level of all detected stars.

From the data obtained with the D90 we extracted light curves of 210 stars; from the 1998 June data 928 stars were processed. The data obtained in 2000 produced light curves of 376 stars. As the observed fields (Fig. 1) overlap, we can search for suspected variability of some stars in independent data sets.

\section{Analysis and results}

In this section we describe the search for new variable stars and analyse the new data on known variables. Frequency analysis was carried out using PERIOD98 (Sperl 1998), which calculates amplitude spectra and offers simultaneous least-squares fits of multiple frequencies, with corresponding amplitudes and phases. Following 
Table 2. New (except for SBL0455) faint variable stars in NGC 6231 based on the deep $V$-data. See the text for discussion of the individual stars. The frequency solutions were obtained to fit our data as well as possible, but are only tentative. "Segg." refers to the numbering system of Seggewiss (1968). $f_{1}-f_{5}$ are detected frequencies (in c $\mathrm{d}^{-1}$ ), $S / N$ is the signal-to-noise ratio of the frequency having the highest (semi-) amplitude ( $a_{V}$, in mmag). The frequency analysis is typically based on 700 to 800 datapoints per star.

\begin{tabular}{crrrrrrrrrrrrr}
\hline SBL & BVF & Segg. & BL95 & $V$ & $B-V$ & $f_{1}$ & $f_{2}$ & $f_{3}$ & $f_{4}$ & $f_{5}$ & $S / N$ & $a_{V}$ & Notes \\
\hline 0306 & & 247 & 14 & 13.987 & 0.668 & 15.6 & 9.8 & 23.8 & & & 5.9 & 6.5 & $\delta$ Sct star \\
0332 & & & & 15.713 & 1.217 & 1.9 & 1.1 & 5.3 & 4.4 & & 69.8 & 57.2 & \\
0417 & 279 & & & 14.618 & 1.154 & 1.1 & & & & & 10.4 & 18.7 & PMS (SBL) \\
0432 & 287 & 84 & & 14.692 & 0.696 & 30.8 & 31.0 & 38.4 & 4.5 & 0.3 & 7.1 & 2.6 & $\delta$ Sct star \\
0455 & 178 & 237 & 41 & 13.455 & 0.611 & 23.8 & 21.8 & 18.9 & & & 15.1 & 10.7 & $\delta$ Sct star (BL95) \\
0508 & 237 & 233 & 50 & 14.120 & 1.082 & 0.3 & 3.1 & 4.7 & & 30.8 & 12.3 & \\
0595 & & 100 & 1 & 14.334 & 0.585 & 41.4 & 36.2 & 41.1 & & 5.5 & 1.6 & $\delta$ Sct star \\
0598 & & 101 & 2 & 13.701 & 0.448 & 3.6 & 1.05 & & & 7.6 & 2.7 & \\
0628 & & 98 & & 15.033 & 1.258 & 1.2 & 3.4 & & & 9.3 & 36.1 & $\gamma$ Dor star? \\
0636 & 324 & & & 15.118 & 0.886 & 1.9 & 4.5 & 8.9 & & 4.7 & 1.6 & \\
0646 & 372 & & & 15.671 & 0.828 & 2.1 & 3.7 & 4.8 & & 14.0 & 4.8 & $\gamma$ Dor star? \\
1949 & 949 & & & 18.28 & 1.55 & 0.67 & 1.01 & & & 23.6 & 111.0 & \\
ASK0171* & & & & 16.2 & & 1.2 & 0.96 & 1.5 & & 9.4 & 48.8 & $\gamma$ Dor star? \\
\hline
\end{tabular}

$*$ Not in SBL; $\alpha_{2000}=3.174, \delta_{2000}=-5.585$ (coordinates in arcminutes relative to $\alpha_{2000}=16^{\mathrm{h}} 54^{\mathrm{m}} 12^{\mathrm{s}}, \delta_{2000}=-41^{\circ} 50^{\prime} 30^{\prime \prime}$, as in SBL).

Breger et al. (1993), we require a peak in the amplitude spectra to have a signal-to-noise $(S / N)$ ratio of at least 4 to be taken into consideration, the noise level being calculated in the residual amplitude spectrum in the region where the peak was found. We are aware that because of our relatively small data sets, the $4 \sigma$ criterion may not be strictly applicable and the suggested frequency solutions are often not unique due to poor spectral windows. The data are, however, sufficiently abundant to serve their purpose: to detect new variables and determine time scales and approximate amplitudes. Only for stars where the frequency solution gave a good fit to the data will this fit be shown.

The cluster colour-magnitude diagram (CMD), based on data from SBL, is shown in Fig. 2. The positions of the newly detected variable stars discussed below are indicated using the identification number of SBL, which we will also use in the discussion of the individual variables. The brightness and colours of the variables, following SBL, are outlined in Tables 2 and 3. These values are not averaged over the pulsational periods found below and may be slightly off depending on the amplitudes of the variations. We note that SBL found an average value of the reddening, $E(B-V)$, of $0.466 \mathrm{mag}$, but values for individual stars could amount to as much as 0.7 magnitudes.

\subsection{The faint stars}

For the faint-star data sets (1998-1999), we visually inspected all light curves for variability and thereby selected stars suitable for further investigation. For these stars we checked their position on the chip (close to a bright star, close to the edge, etc.), calculated amplitude spectra and compared overlapping data sets. Only stars for which the evidence for intrinsic variability was found

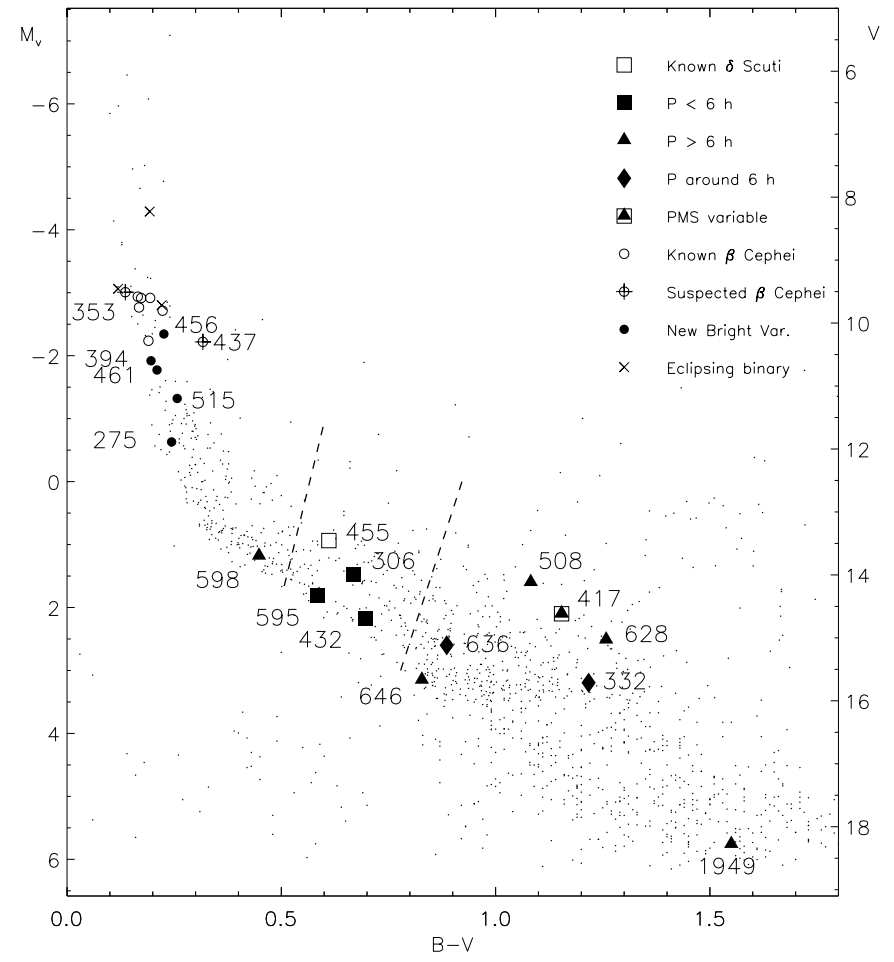

Fig. 2. The colour-magnitude diagram of NGC 6231, based on data from SBL. The positions of the detected variable stars are indicated. The dashed line is the $\delta$ Scuti instability strip from Breger (2000). The $\gamma$ Doradus stars are expected to be found near the cool border of the $\delta$ Scuti instability strip (Handler 1999), while the SPB and $\beta$ Cephei stars are found among stars brighter than $V=12$.

convincing (and significant on the $4 \sigma$ level) were taken as being variable and will be discussed below. For some of these stars, the evidence for variability is also present 
Table 3. New and confirmed bright variable stars in NGC6231, based on the 2000-data. Tentative frequency solutions are given, see Table 2 for explanations of the entries. $a_{b}$ is the (semi-) amplitude of the strongest frequency in the $b$-band.

\begin{tabular}{rrrrrrrrrrrrrrr}
\hline SBL & BVF & Segg. & BL95 & $V$ & $B-V$ & $f_{1}$ & $f_{2}$ & $f_{3}$ & $f_{4}$ & $f_{5}$ & $f_{6}$ & $S / N$ & $a_{b}$ & Notes \\
\hline 0226 & 20 & 253 & 31 & 9.580 & 0.165 & 14.9 & 1.7 & 4.5 & & & & 13.8 & 6.9 & $\beta$ Cep star \\
0268 & 26 & 282 & 158 & 9.752 & 0.168 & 8.4 & 2.6 & & & & & 6.9 & 3.1 & $\beta$ Cep star \\
0275 & & 249 & 19 & 11.888 & 0.244 & 1.65 & 0.74 & & & & & 14.2 & 12.8 & new SPB? \\
0303 & 37 & 261 & 67 & 10.281 & 0.190 & 10.1 & 11.0 & 1.3 & 11.5 & 0.2 & 12.7 & 22.4 & 10.1 & $\beta$ Cep star \\
0394 & 39 & 209 & & 10.599 & 0.196 & 1.4 & 1.7 & & & & & 8.1 & 5.5 & new SPB? \\
0456 & 36 & 104 & 127 & 10.174 & 0.226 & 3.1 & 3.9 & 0.1 & & & & 10.4 & 7.7 & new $\beta$ Cep star \\
0461 & 40 & $294 \mathrm{~A}$ & 89 & 10.745 & 0.210 & 0.4 & 0.5 & & & & & 10.4 & 5.9 & new SPB? \\
0486 & 22 & 238 & 45 & 9.603 & 0.173 & 0.4 & 0.3 & 14.6 & 13.8 & 10.4 & & 12.3 & 3.2 & $\beta$ Cep star \\
0515 & 49 & & 129 & 11.198 & 0.257 & 5.3 & 3.5 & 0.8 & 7.4 & & & 7.1 & 4.7 & new $\beta$ Cep star? \\
0653 & 28 & 110 & 11 & 9.803 & 0.223 & 8.3 & 0.9 & & & & & 6.0 & 4.0 & $\beta$ Cep star \\
0712 & 23 & 150 & 124 & 9.601 & 0.194 & 9.9 & 8.3 & 10.6 & & & & 11.2 & 6.0 & $\beta$ Cep star \\
\hline
\end{tabular}

in the 2000-data, although these light curves have much higher noise levels (as they were optimised for the bright stars) and poorer sampling as a result of the acquisition of four-colour photometry - as illustrated in Fig. 3.

The detected faint variable stars, along with preliminary frequency solutions, are presented in Table 2. In Figs. 4-16 we show amplitude spectra and light curves for the variables. Of the light curves, we only show enough data to support our claim of variability, the amount depending on the time scales of the variations. For each variable star we briefly discuss the observed variations and, when possible, give a preliminary classification. However, data obtained more recently will lead to a more precise and definite classification and will be included in forthcoming papers.

One result from BVF is of great interest here. BVF suggest that all cluster stars between $14^{\mathrm{m}}$ and $16^{\mathrm{m}}$ are PMS stars. This is based on the fact that basically all stars in this magnitude range are found above the ZAMS, without following its shape. It is exactly in this magnitude range that we detect our new faint variable stars. We will, therefore, be cautious in discussing the evolutionary status of these stars, which should all be investigated in further detail - including determination of cluster membership. We will now discuss the new faint variable stars individually.

SBL0306 is a short-period variable star with an amplitude of a few mmag. The variability is clearly visible in the light curves (Fig. 4), but a part of the data is contaminated by bleeding from bright stars. The data are not sufficiently abundant to pin down a good frequency solution and the frequencies given in Table 2 do not describe the light curves very well. One frequency $\left(9.8 \mathrm{c} \mathrm{d}^{-1}\right)$, however, is recovered in the 2000-data. The star is situated right in the $\delta$ Scuti instability strip and this, along with the periods and amplitudes, leads us to suggest that it is indeed a $\delta$ Scuti star.

SBL0332 is also clearly variable, with periods of the order of days and amplitudes of several hundredths of a magnitude. It is found at the cool side of the $\delta$ Scuti instability strip, but further to the red than expected for a

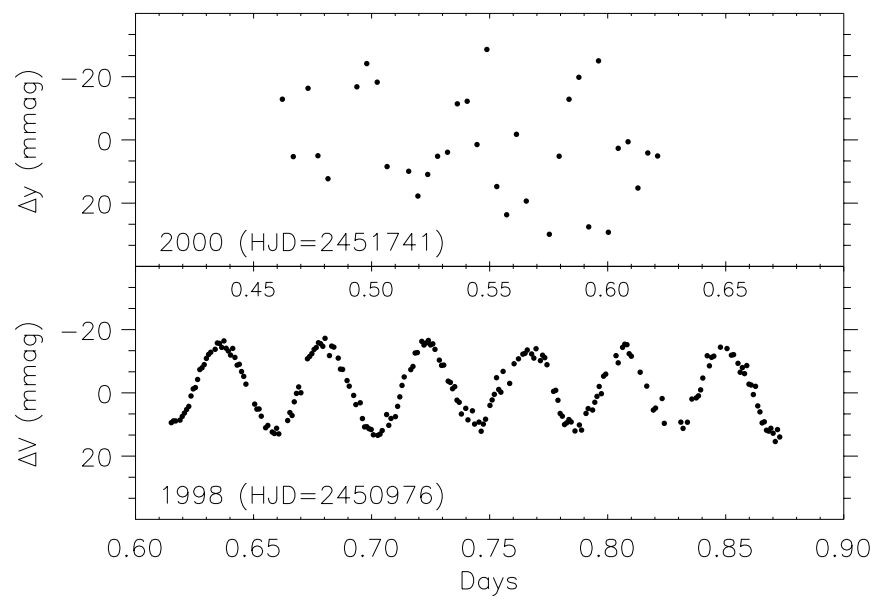

Fig. 3. Comparison between the deep 1998 data (lower panel) and the 2000-data for one of the faint variable stars (SBL0455).
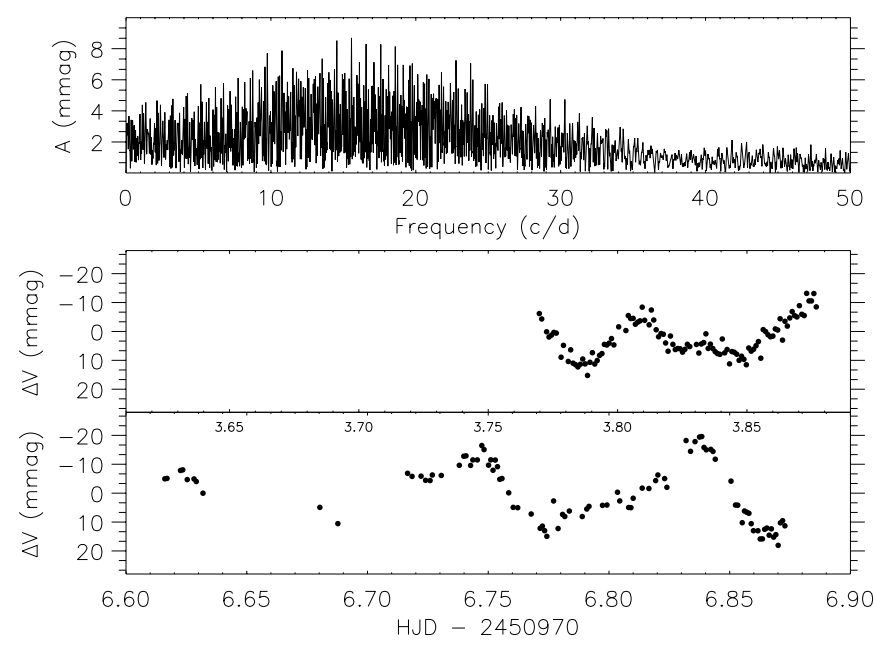

Fig. 4. Amplitude spectrum and $V$ light curves of SBL0306. Data were obtained during 8 nights, of which 2 are shown.

$\gamma$ Doradus star. It could, in principle, be a $\gamma$ Doradus star affected by intracluster differential reddening, but two frequencies (above $4 \mathrm{~cd}^{-1}$ ) are higher than what would be expected for a $\gamma$ Doradus star (below about $3 \mathrm{~cd}^{-1}$, see e.g. Kaye et al. 1999). Four frequencies describe the variations 

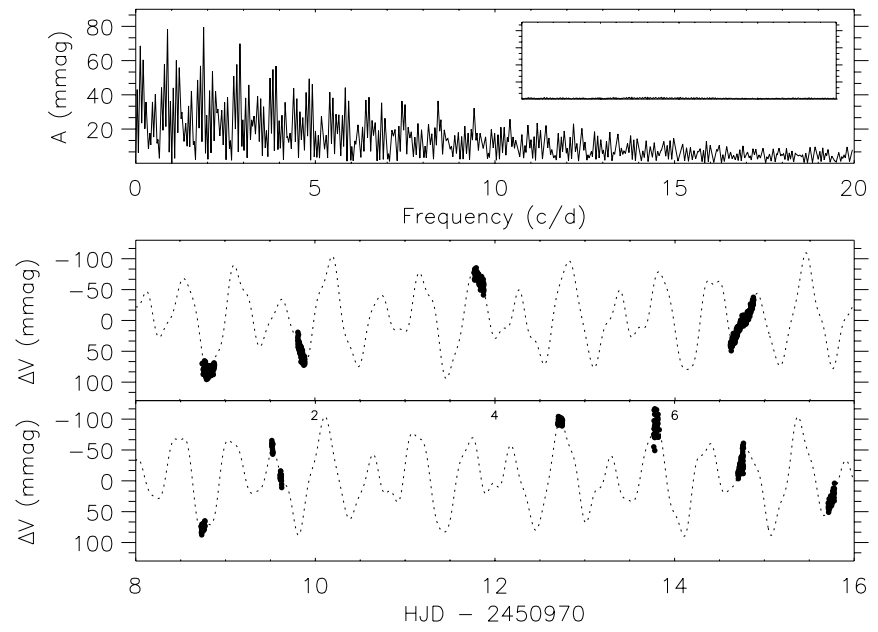

Fig. 5. Amplitude spectrum and $V$ light curves of SBL0332. The residual amplitude spectrum after pre-whitening with four frequencies is shown, on the same scale, in the insert in the upper panel. All data are shown.
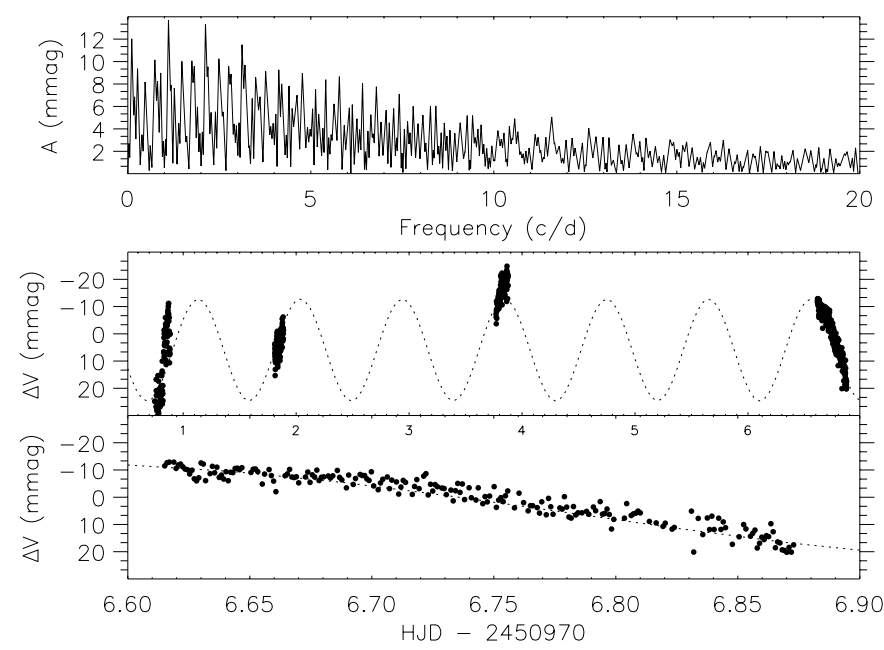

Fig. 6. Amplitude spectrum and $V$ light curves of SBL0417 a variable PMS star. Four out of ten nights of data are shown.

well and the residual amplitude spectrum, shown as the insert in Fig. 5, is flat after pre-whitening with the four terms and the residual noise level is less than 1 mmag.

SBL0417 was found to be a PMS star by SBL and we find that it is also variable. The variability is displayed in Fig. 6. Only one frequency can be found, but it does not describe the variability completely. If the variations are caused by pulsations, it could be a PMS $\gamma$ Doradus star, given the time scale of the variations of about a day. As this is known to be a PMS star, the variations may be irregular, but the present data set is not sufficiently abundant to determine this.

SBL0432 is a short-period variable showing effects of beating between several frequencies (Fig. 7). The periodogram does not yield a clear-cut frequency solution, but indicates, along with the time series, that the variations occur on two time scales. As given in Table 2, we find three frequencies in the region $30-40 \mathrm{~cd}^{-1}$ and two
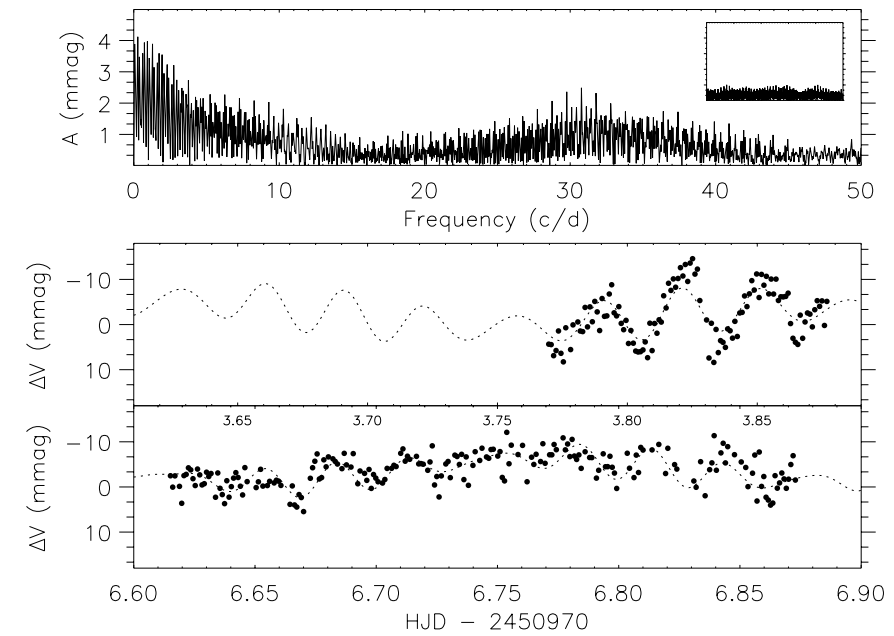

Fig. 7. Amplitude spectrum and $V$ light curves of SBL0432. The residual amplitude spectrum after subtracting the 5 terms in Table 2 is shown, on the same scale, as the insert in the upper panel. Two out of nine nights of data are shown.
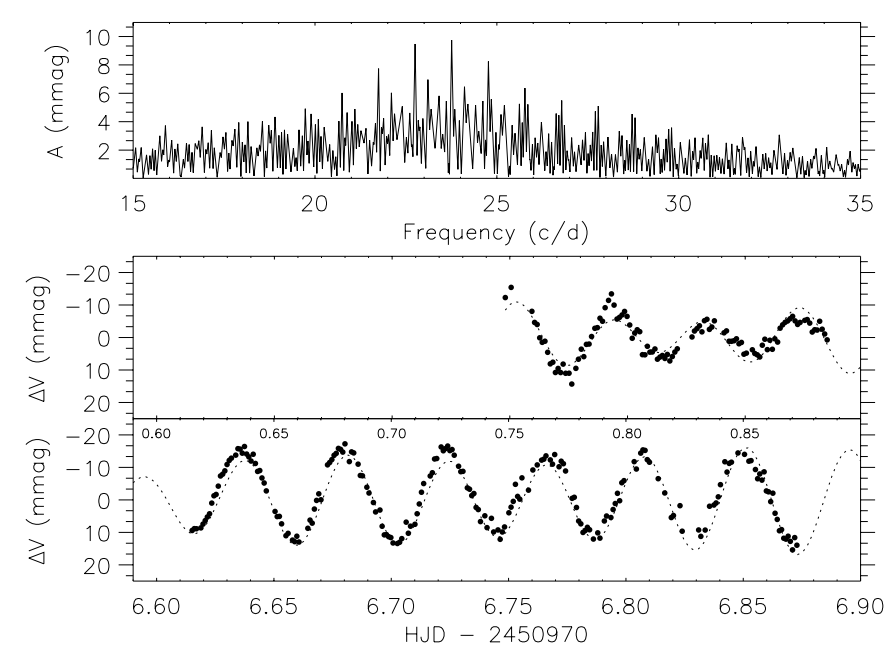

Fig. 8. Amplitude spectrum and $V$ light curves of the $\delta$ Scuti star SBL0455. Two out of nine nights of data are shown.

below $5 \mathrm{~cd}^{-1}$. The star is found near the main sequence, in the $\delta$ Scuti instability strip, and the evidence that it is a $\delta$ Scuti star is rather convincing - especially considering the beating light curve, typical for a low-amplitude $\delta$ Scuti star. The additional presence of low-frequency variations makes it a very interesting object.

SBL0455 was suggested to be a $\delta$ Scuti star in BL95. This is very clearly confirmed by our data, as shown in Fig. 8. The light curves suggest the presence of several frequencies (beating), which is confirmed by the frequency analysis. We find 3 frequencies, but more are probably present. The amplitude of the frequency at $18.9 \mathrm{~cd}^{-1}$ apparently increased from $3.1 \mathrm{mmag}$ in 1998 to $10.7 \mathrm{mmag}$ in 2000, although this result should be taken with caution because of the much lower quality for the fainter stars in the 2000 data set. The star is positioned off the main sequence in the CMD: if it is a single star and a member of the cluster, it is a PMS star. 

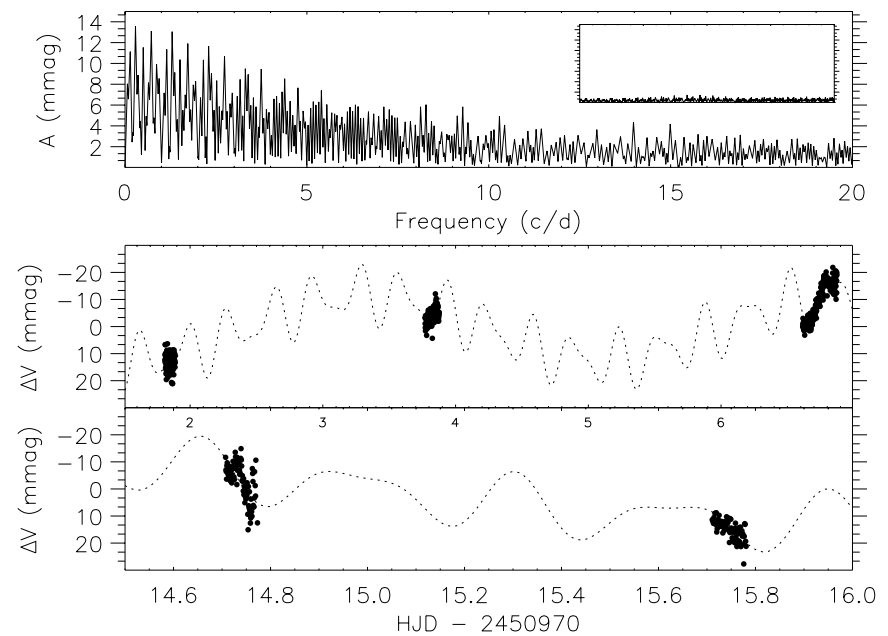

Fig. 9. Amplitude spectrum and $V$ light curves of SBL0508. Five out of nine nights of data are shown. The insert shows the residual amplitude spectrum, on the same scale, after subtracting three frequencies.
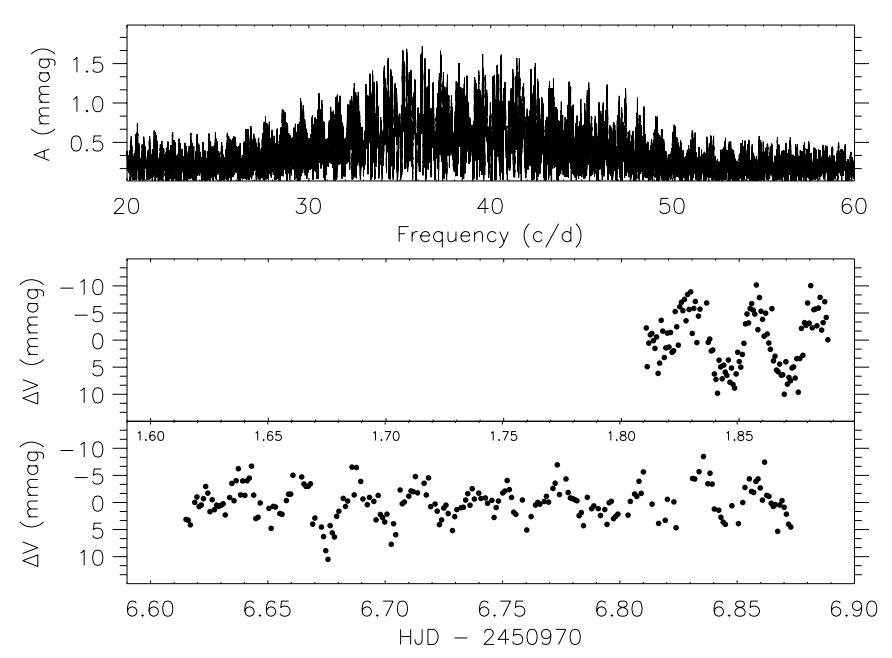

Fig. 10. Amplitude spectrum and $V$ light curves of SBL0595. Two out of 19 nights of data are shown.

SBL0508 is variable (Fig. 9) on two time scales: about 4 days and 6-8 hours. The star is positioned to the cool side of the $\delta$ Scuti instability strip, off the main sequence. We find 3 frequencies with amplitudes between 4 and $12 \mathrm{mmag}$, but more data are needed on this object.

SBL0595 is a short-period variable with a typical $\delta$ Scuti type light curve. It is also found in the $\delta$ Scuti instability strip and it most likely belongs to this class. The amplitude spectrum is complicated and the light curve is somewhat noisy (Fig. 10) compared to the low amplitude of the variations. The variability is clearly present, but can be represented by several different frequency solutions. We find 3 frequencies near $40 \mathrm{~cd}^{-1}$, but these merely indicate the time scale of the variations.

SBL0598 shows relatively long-period variations (in the $\gamma$ Doradus range) and amplitudes of only a few mmag. Some of the light curves can be seen in Fig. 11. We find two frequencies which describe the light curve reasonably
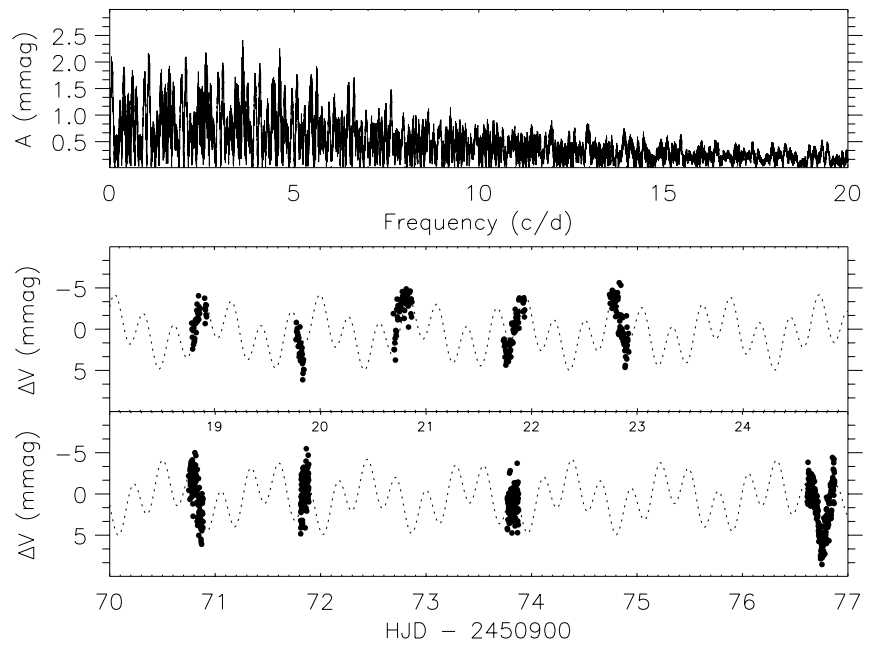

Fig. 11. Amplitude spectrum and $V$ light curves of SBL0598. Note the low amplitude of the variations. Data were obtained on 20 nights, of which nine are shown.

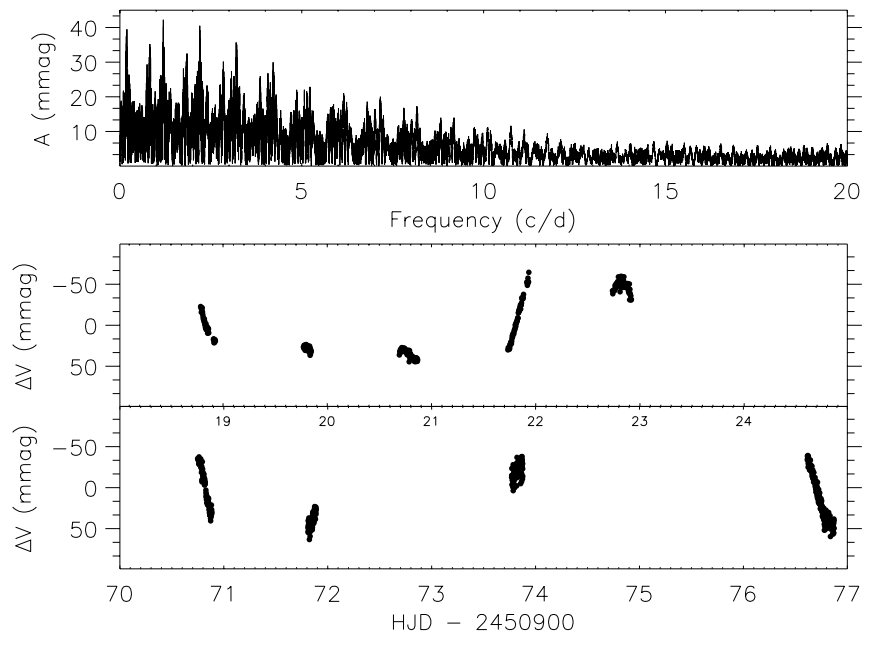

Fig. 12. Amplitude spectrum and $V$ light curves of SBL0628. Data were obtained on 20 nights, of which 9 are shown.

well. The star is positioned to the hot/blue side of the $\delta$ Scuti instability strip and it is probably not a cluster member, even given the significant amount of differential reddening present over the cluster (see SBL). SBL0598 is SAAO 2 - BL95 labels the foreground $\delta$ Scuti star with this number, but that bright foreground object is in fact SAAO 3.

SBL0628 shows variability on a time scale of days and with relatively high amplitude (Fig. 12). We attempted to fit the light curves with two frequencies, but the fit deviates from the light curves on several nights. The dominant frequency $\left(1.2 \mathrm{c} \mathrm{d}^{-1}\right)$ is present in the D90 and D154 data individually, while for the secondary frequency two different aliases are highest in the two data sets. This star could be a $\gamma$ Doradus star, also considering its position in the CMD.

SBL0636 is variable on a very low level. Figure 13 shows that mmag variations are present, both in the data obtained with the D90 (middle panel) and with the D154 


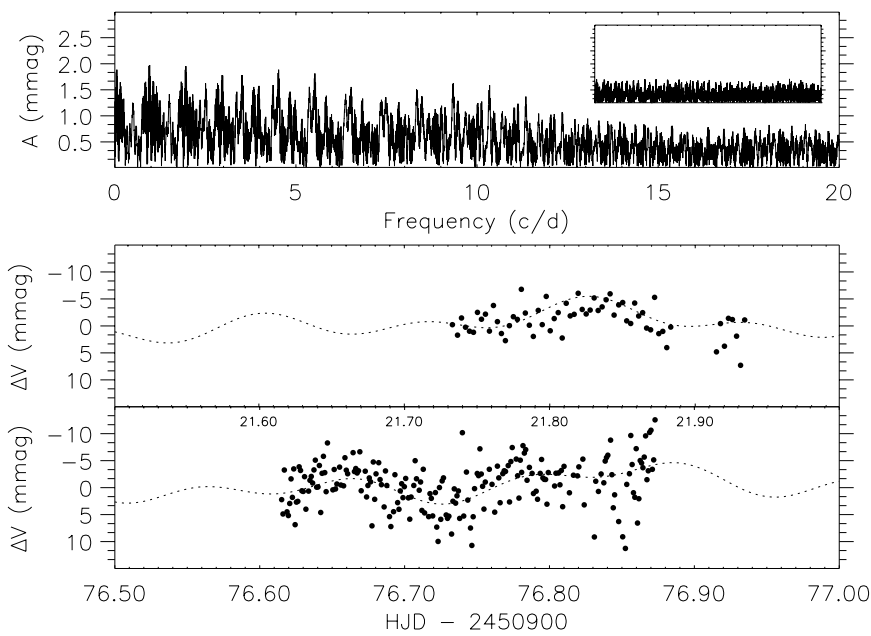

Fig. 13. Amplitude spectrum and $V$ light curves of SBL0636. The insert shows the pre-whitened data ( 3 frequencies) on the same scale. Data were obtained during 19 nights of which 2 are shown (in the upper panel one from the D90, in the lower panel one from the D154)
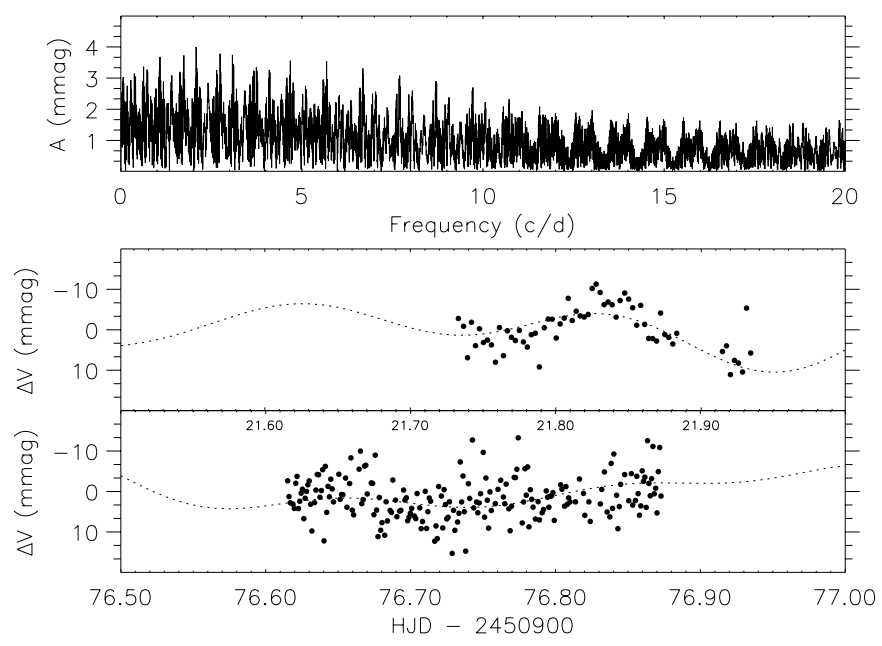

Fig. 14. Amplitude spectrum and $V$ light curves of SBL0646. Useful data were obtained during 14 nights, of which 2 are shown.

(lower panel). In the amplitude spectrum, the variations are not obvious, but fitting 3 frequencies ranging from about 2 to $9 \mathrm{~cd}^{-1}$ leaves a clean residual in the amplitude spectrum, and the frequency solution seems to fit the light curves rather well. The star is situated in the area of the CMD where the $\gamma$ Doradus stars are expected to be, but only one of the frequencies is in the range expected for the $\gamma$ Doradus stars.

SBL0646 is very similar to SBL0636 with regard to amplitudes and frequencies of the variations. Both stars are also situated close to each other in the CMD. Light curves and the amplitude spectrum can be seen in Fig. 14. The middle panel has data from the D90, the lower panel data from the D154. We find 3 frequencies to be present between 2 and $4 \mathrm{~cd}^{-1}$. This star might be a $\gamma$ Doradus star.
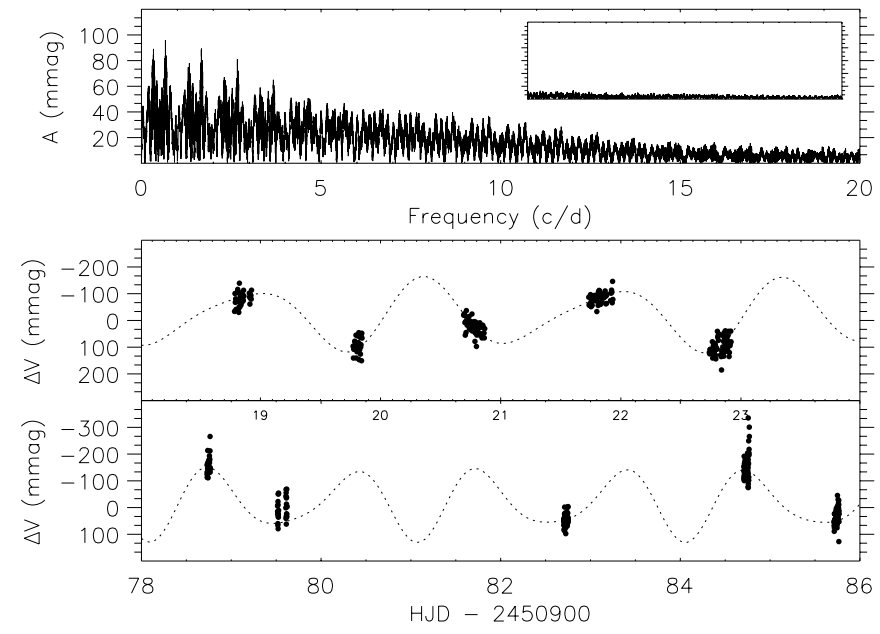

Fig. 15. Amplitude spectrum and $V$ light curves of SBL1949. The insert shows the amplitude spectrum, on the same scale, after subtracting 2 frequencies. Data were obtained during 18 nights of which 10 are shown.

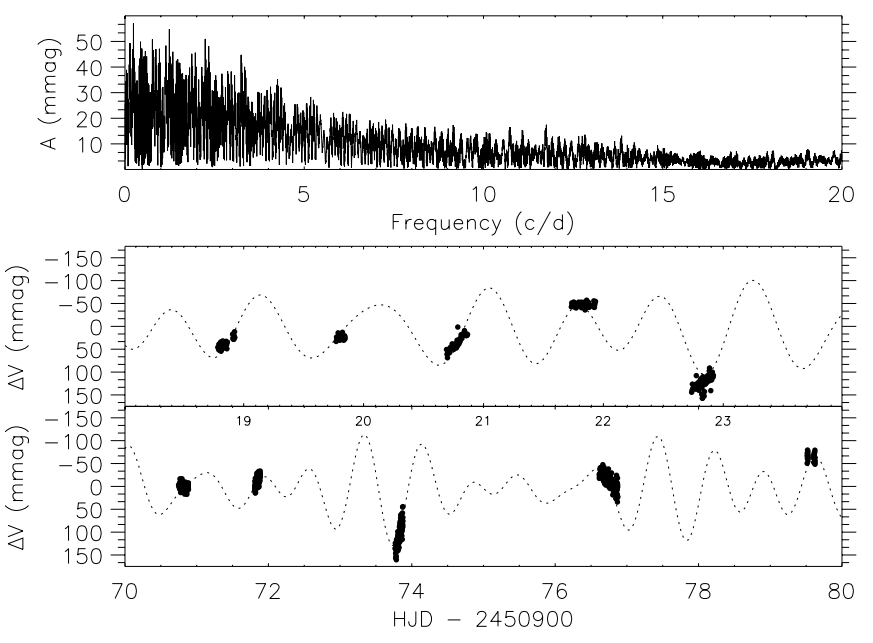

Fig. 16. Amplitude spectrum and $V$ light curves of ASK0171. Data were obtained during 16 nights of which 10 are shown.

SBL1949 is a very faint star $(V=18.28)$, but slow variations on a time scale of days are clearly present in the light curves, resulting in low-frequency peaks in the amplitude spectrum (Fig. 15). Two frequencies describe both the amplitude spectrum and the light curves fairly well.

ASK0171 is clearly variable, but was not observed by SBL or BVF. The variations occur on a time scale of a day. We have no colour information on this star, but the $V$-magnitude is approximately $16^{\mathrm{m}}$. It may be a $\gamma$ Doradus star based on the periods and the brightness, but colour data are clearly needed on this object. The star is too faint to have reliable photometry in the 2000 uvby data sets.

Positions of all the faint variable stars in the colourmagnitude and colour-colour diagrams presented by SBL were checked using their photometry. Only the PMS star SBL0417 deviates from the majority of stars in these diagrams. 

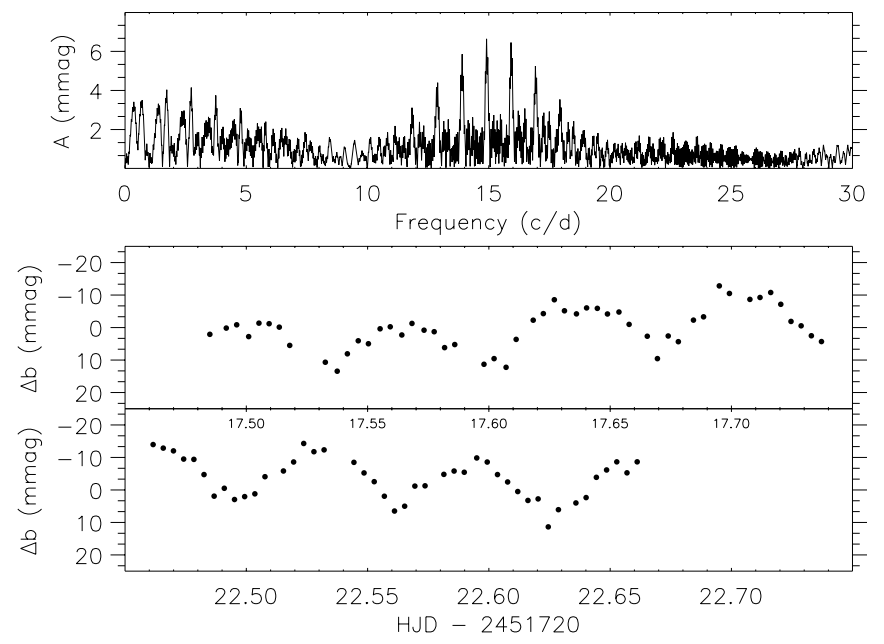

Fig. 17. Amplitude spectrum and $b$ light curves of SBL0226.

\subsection{The bright stars}

For the 2000-data optimised for the ninth magnitude stars, uvby photometry is available. One night from 2000 June yielded data of comparatively low quality and has been rejected. For the stars discussed below, there are thus 8 nights of data available in the analysis, covering in total about 300 datapoints per filter. In order to search for new variable stars, we visually inspected the light curves and calculated amplitude spectra for the different bands. A star was deemed worthy of further investigation if signs of variability could be seen in the light curves from the individual filters and/or when there was excess power at the same frequencies in the corresponding amplitude spectra. The relatively small amount of data resulted in a poor spectral window (which can be assessed from the dominant frequency near $15 \mathrm{c} \mathrm{d}^{-1}$ in the upper panel of Fig. 17) and the suggested frequency solutions, given in Table 3 for the stars discussed below, should again be regarded as preliminary. All the stars discussed below, except SBL0275 for which no membership information is available, were classified as likely members of the cluster by BVF (from whom we have also adopted the spectral classifications). For the known $\beta$ Cephei stars we show, as an example, data for a single star only. For several of those stars, we find evidence for long-period variations superimposed on the faster $\beta$ Cephei pulsations. Such variations, which were also noticed by BS83, may well be real and should be investigated in further detail when more data are available. SBL0226 (V945 Sco, B1 V + B1 V) is a known $\beta$ Cephei variable and double-lined binary. We find three frequencies, of which the first and an alias of the third, match the two corresponding frequencies in BS83. However, these frequencies do not describe the light curves completely. The amplitude spectrum and examples of light curves, representative for the quality of data on the other known $\beta$ Cephei stars as well, are shown in Fig. 17.

SBL0268 (B2 V + B2 V) is also a known $\beta$ Cephei variable and a double-lined binary. We find two frequencies, one of which is the same as in BE85. Variations at higher
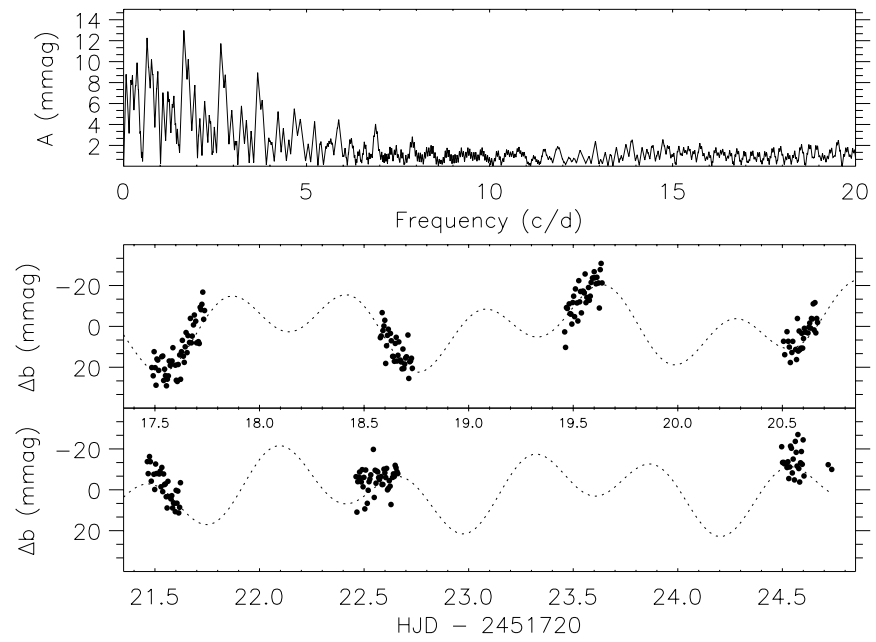

Fig. 18. Amplitude spectrum and $b$ light curves of SBL0275.
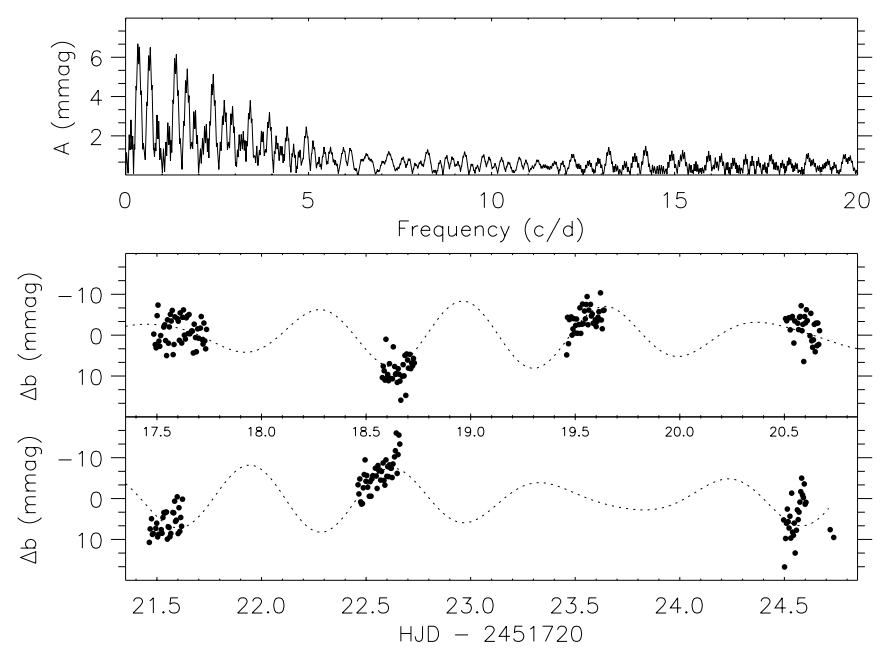

Fig. 19. Amplitude spectrum and $b$ light curves of SBL0394.

frequencies are visible in all four filters, but are not detected in our data because of a too low $S / N$.

SBL0275 is a new SPB candidate. The star is situated very close to the ZAMS, possibly inside the SPB instability strip (to be verified at a later stage). The detected low-frequency variations are consistent with such a classification (see e.g. Pamyatnykh 1999). The evidence for the variability is shown in Fig. 18.

SBL0303 (B2 IV) is a known $\beta$ Cephei variable and probable binary (García \& Mermilliod 2001). It has several frequencies around $10 \mathrm{~cd}^{-1}$, and of the five we detect in that region, four correspond to the frequencies (or their $1 \mathrm{~cd}^{-1}$ aliases) from BS83. We find two peaks in the lowfrequency region, of which one is close to a $1 \mathrm{~cd}^{-1}$ alias of the single low-frequency found by BS83.

SBL0394 (B2 IV-V) is a new SPB candidate. The lowfrequency variation is clearly seen in Fig. 19, but aliases of the two frequencies listed in Table 3 can also fit the variation.

SBL0456 is, based on the position in the CMD and the shape of the light curves (Fig. 20), a new $\beta$ Cephei candidate. The frequency solution does not match the light 


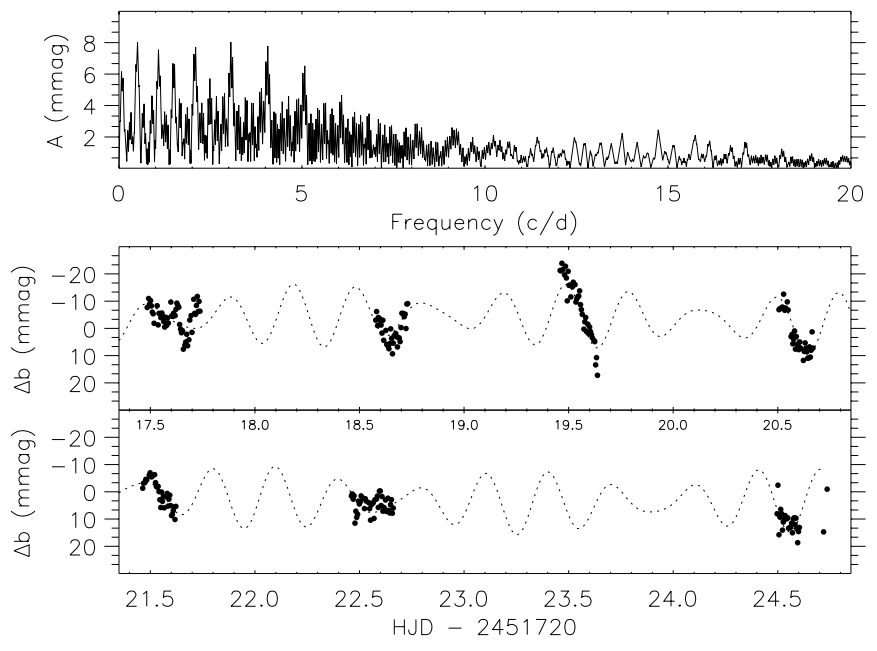

Fig. 20. Amplitude spectrum and $b$ light curves of SBL0456.

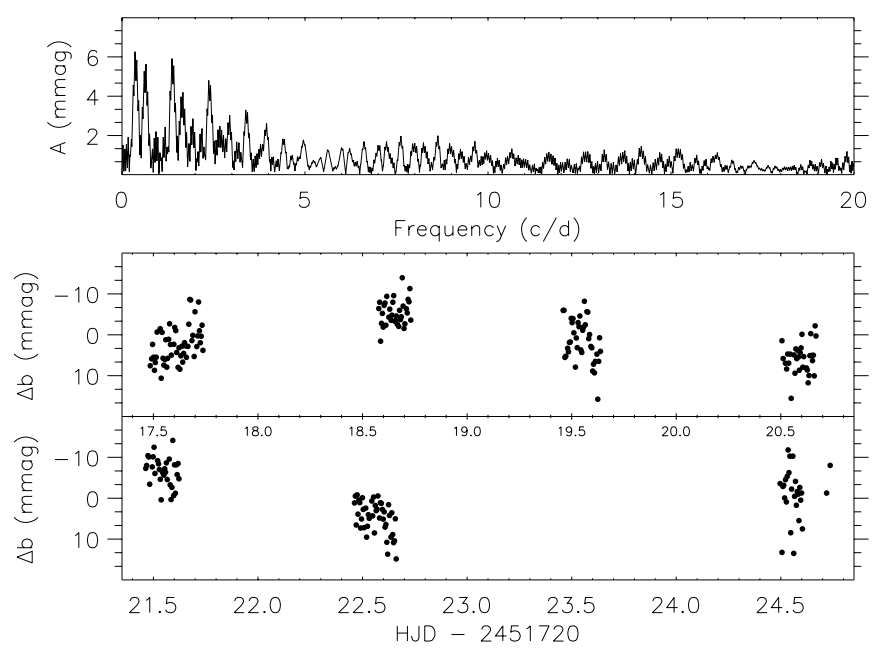

Fig. 21. Amplitude spectrum and $b$ light curves of SBL0461.

curves completely; variations on a shorter time-scale than those listed in Table 3 may be present in the light curves, but the data presently available are insufficient to detect such variations.

SBL0461 (B1.5 V) is a new SPB candidate. The longperiod variations are visible in the light curves in Fig. 21, but should be checked with new and more abundant data. SBL0486 (V964 Sco, B1 V(n)) is a known $\beta$ Cephei variable; BE85 find the three frequencies 11.3835, 14.7983 and $18.0741 \mathrm{c} \mathrm{d}^{-1}$, of which we find aliases of the two first. We also find evidence for two low-frequency variations with periods around 3 days.

SBL0515 $(\mathrm{B} 1 \mathrm{~V}(\mathrm{n}))$ is variable with several frequencies in the range $3-8 \mathrm{c} \mathrm{d}^{-1}$, but aliasing gives several possible frequency combinations. The frequencies and amplitudes suggest a $\beta$ Cephei classification, but this seems inconsistent with the position of the star in the CMD, as it is much fainter than the other $\beta$ Cephei stars. This could suggest that it is a non-cluster member, although BVF find it to be a likely member. It is a very interesting target, but new data are clearly needed.
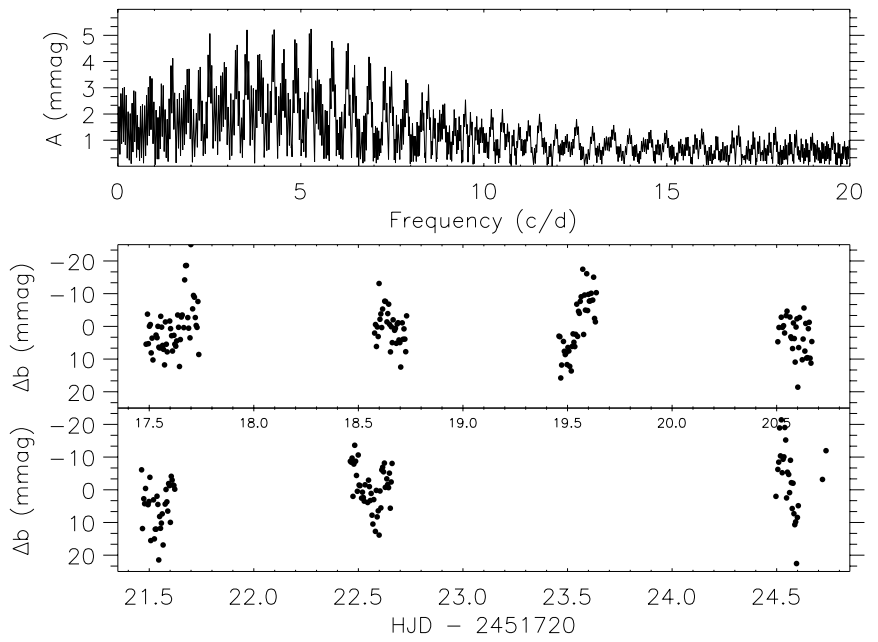

Fig. 22. Amplitude spectrum and $b$ light curves of SBL0515.

SBL0653 (B1 V) is a known $\beta$ Cephei variable. We find an alias of the BS83 oscillation frequency at $9.27 \mathrm{~cd}^{-1}$. The low frequency variation at $0.9 \mathrm{c} \mathrm{d}^{-1}$ is not stable: if we prewhiten using aliases to the $8.3 \mathrm{c} \mathrm{d}^{-1}$ frequency, the highest low-frequency peak is no longer located at $0.9 \mathrm{~cd}^{-1}$ or aliases to this value. There is no indication of an oscillation at $16.4 \mathrm{~cd}^{-1}$ as found by BS83.

SBL0712 (V920Sco, B1 V(n)) is a known $\beta$ Cephei variable with 3-4 frequencies found around $9 \mathrm{~cd}^{-1}$. A lowfrequency variation of $1.69 \mathrm{~cd}^{-1}(14.2 \mathrm{~h})$ appears to be present just below the detection threshold, similar to what was found in BS83 (16.63 \pm 0.12 h). García \& Mermilliod (2001) list the star as a possible binary.

\subsection{Possible variable stars and eclipsing binaries}

Two possible $\beta$ Cephei stars from BE85 were in the field of our 2000-observations. In both cases variability is seen, but detections cannot be made at a significant level.

SBL0353 (S289, SB1) shows variability in the frequency region $10-15 \mathrm{~cd}^{-1}$, but the amplitudes are below one mmag (b) and are not significant in our data. It is a possible $\beta$ Cephei star according to BE85, but more data are needed to confirm this. Similar variations are seen in all four filters, but in all cases with amplitudes below the detection threshold. If the variation is periodic it is in the frequency range 10 to $15 \mathrm{~cd}^{-1}$ with amplitudes up to $1 \mathrm{mmag}$.

SBL0437 has a possible oscillation near $15 \mathrm{c} \mathrm{d}^{-1}$ and perhaps also variations at low frequencies. It is a possible $\beta$ Cephei star according to BE85, but although our data confirm that variability may be present, the data currently available do not allow a detection at the $4 \sigma$ level in the amplitude spectrum.

No new detections of eclipsing binary systems result from the data, but parts of eclipses were observed for two known EBs.

SBL0505 (S224, SAAO100) is an eclipsing O9 IV binary (Levato \& Morrell 1983, BL95) for which 
García \& Mermilliod (2001) determine new orbital elements $(P=2.453087 \mathrm{~d})$. We observed a rising and a descending branch of two individual minima, but could not determine the times of mid-eclipse from the data.

SBL0521 (S232, SAAO040) is a single-lined eclipsing binary $(\mathrm{B} 0.5 \mathrm{~V})$ observed spectroscopically by Levato \& Morrell (1983) and García \& Mermilliod (2001) and photometrically by BL95. We observed a part of an eclipse with an 0.15 minimum at HJD 2451740.6702. The full primary eclipse is estimated to last more than 8 hours. A weaker minimum of 0.05 on JD 2449500.51 was reported by BL95. The phase coverage is still insufficient to determine a period.

SBL0334 (S272, SAAO105) is a possible eclipsing binary for which BL95 observed a single sharp minimum of $0.05 \mathrm{mag}$. Their suggested period of $1.6 \mathrm{~d}$ is unlikely, as we have better than $90 \%$ phase coverage for that period, but see no eclipses. A modulation of the light curve may be present, but the currently-available data are inconclusive.

\section{Conclusions}

We have searched for and detected new variable stars in NGC 6231. The available data allowed us to determine variability and derive preliminary frequency solutions as estimates of the time scales and amplitudes of the variations. We found 17 new variable stars of which, among the fainter stars, three are $\delta$ Scuti stars (SBL0306, SBL0432 and SBL595) and three are $\gamma$ Doradus candidates (SBL0628, SBL0646 and ASK0171). Among the brighter stars, one or two are new $\beta$ Cephei candidates (SBL0456, SBL0515) while three stars are SPB candidates (SBL0275, SBL0394 and SBL0461). One variable, SBL0417, is a PMS star according to SBL. For 6 stars, no good classification could be suggested based on the present data (SBL0332, SBL0417, SBL0508, SBL0598, SBL0636 and SBL1949).

We have furthermore discussed new data on a known $\delta$ Scuti star (SBL0455), where we were able to confirm the variability, and six known $\beta$ Cephei stars (SBL0226, SBL0268, SBL0303, SBL0486, SBL0653 and SBL0712). For two suspected $\beta$ Cephei stars, SBL0353 and SBL0437 (BE85), we also suspect variability but need more data before a definite detection can be made. We observed incomplete eclipses for two EBs (SBL0505 and SBL0521).

Acknowledgements. T.A., C.S. and M.R.K. acknowledge financial support from the Belgian Fund for Scientific Research (FWO). This project was supported by the Flemish Ministry for Foreign Policy, European Affairs, Science and Technology, under contract BIL 99/2 and by the Danish Natural Science Research Council through the centre for Ground-Based Observational Astronomy. Our research has made use of the SIMBAD database operated at CDS, Strasbourg, France, the NASA Astrophysics Data System and the STSci Digitized sky survey.

\section{References}

Arentoft, T., \& Sterken, C. 2000, J. Astron. Data, 6, 4c Balona, L. A., \& Shobbrook, R. R. 1983, MNRAS, 205, 309 (BS83)

Balona, L. A., \& Engelbrecht, C. A. 1985, MNRAS, 212, 889 (BE85)

Balona, L. A., \& Laney, C. D. 1995, MNRAS, 276, 627 (BL95)

Baume, G., Vázquez, R. A., \& Feinstein, A. 1999, A\&AS, 137, 233 (BVF)

Breger, M., Stich, J., Garrido, R., et al. 1993, A\&A, 271, 482

Breger, M. 2000, in Proc. of Delta Scuti and Related Stars, Vienna 4-7 August 1999, ASP Conf. Ser., 210, 3

Cousins, A. W., \& Warren, P. R. 1963, MNASSA, 22, 65

Freyhammer, L. M., Andersen, M. I., Arentoft, T., et al. 2002, in preparation

García, B., \& Mermilliod, J. C. 2001, A\&A, 368, 122

Handler, G. 1999, MNRAS, 309, L19

Kaye, A. B., Handler, G., Krisciunas, K., et al. 1999, PASP, 111,840

Kjeldsen, H., \& Frandsen, S. 1992, PASP, 104, 413

Levato, H., \& Morrell, N. 1983, ApJ, 23, L183

Pamyatnykh, A. A. 1999, Acta Astron., 49, 119

Raboud, E. 1996, A\&A, 315, 384

Seggewiss, W. 1968, Veröff. Astron. Inst. Univ. Bonn, 79

Sperl, M. 1998, Comm. in Asteroseismol., 111, 1

Sterken, C., Knudsen, M. R., Duerbeck, H. W., et al. 2002, in Observational aspects of pulsating B- and A stars, ed. C. Sterken \& D. W. Kurtz, ASP Conf. Ser., in press

Sung, H., Bessell, M. S., \& Lee, S.-W. 1998, AJ, 115, 734 (SBL) 